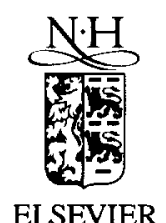

ELSEVIER

\title{
Biaxial Kerr magnetometry in oblique field for the study of thin films with a perpendicular anisotropy
}

\author{
Wim Geerts, Cock Lodder *, Theo Popma \\ Mesa Research Institute, University of Twente, PO Box 217, 7500 AE Enschede, The Netherlands
}

Received 5 July 1993; in revised form 24 January 1994

\begin{abstract}
A measurement system based on the magneto-optic Kerr effect was developed in order to study magnetic thin films for applications in recording media. The sample can be rotated in the magnetic field by using two Al mirrors. Two components of the magnetization can be measured for different directions and magnitudes of the applied field. The measurement performance was shown by experiments on sputtered $\mathrm{Co}-\mathrm{Cr}$ films. The results are in agreement with those of biaxial VSM experiments performed on the same sample. Preliminary results suggest that rotation of the spins, which mainly occur for larger fields, oppose the reversal of the magnetization.
\end{abstract}

\section{Introduction: recording}

During the last three decades the information density in magnetic recording materials has increased by more than a factor of 10000 . The availability/application of better technologies with which thinner media and smaller head gaps could be produced have greatly reduced the price per bit. In future it can be expected that other product features, particularly compactness and stability, will become important reasons for the development of new media.

Depending on the orientation of the magnetization in the medium, several types of recording methods can be considered. In the conventional longitudinal recording mode, the medium is magnetized parallel to the film surface and parallel to the track (see Fig. 1a). In the perpendicular recording mode [1], the bits are written perpendicular to the film surface (see Fig. 1b). Although this mode should be energetically more favourable than the longitudinal one, Mallinson

\footnotetext{
* Corresponding author.
}

showed that at the surface of both kinds of recording media no major differences exist [2]. The main advantage of the perpendicular mode is the possibility of using a soft magnetic underlayer (see Fig. 1c). This layer acts as a mirror in the writing process and will partly compensate the magnetic charges at the bottom side of the recording layer in the reading process. In spite of the larger information densities that have been obtained in perpendicular recording media, worldwide application has held up until now. Investments in new production facilities and for the development of new heads are at present most probably expected to be too large to be compensated by the lower prices of both the medium and the head.

Recently the so-called oblique recording mode (see Fig. 1d) has gained enhanced interest. In this case the medium consists of oblique evaporated media [3] in which the anisotropy angle is non-perpendicular to the surface. This technique combines the advantage of the sharp bit transitions of perpendicular media with the possibility of using a conventional ring-type head. Conventional scalar measure- 
a

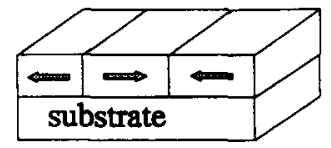

c

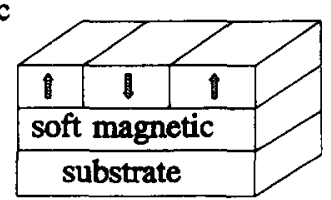

b

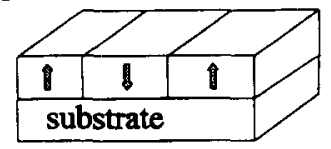

d

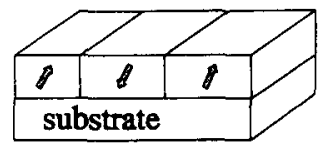

Fig. 1. Recording modes: (a) longitudinal, (b) perpendicular, (c) perpendicular with underlayer, and (d) oblique (for example, metal evaporated tape).

ment techniques do not supply enough information for the investigation of these so-called oblique-deposited films.

High-density recording is not a process for which the field is neatly applied along the easy magnetization direction of the medium. The vectorial character of the recording process is illustrated in Fig. 2, which shows the amplitude and the direction of the head field versus the time as experienced by the magnetic film. At $t=0$ the single-pole-type head is just above the point of observation. At the top of the graph the vector of the head-field is given for several time points. In order to understand the complex recording process it is necessary to know the angle dependence of the magnetic reversal process. Bernards et al. simulated the recording process successfully by performing field-angle-dependent measurements on several media using a biaxial VSM [4].

Hence both the investigation of the recording process as well as the investigation of oblique media require a vectorial approach. Several measurement techniques have been extended in order to be able to perform field-angle-dependent measurements; indirect techniques as the magneto-optic (MO) Kerr [5] and anomalous Hall [6], as well as the direct VSM technique [7].

In this paper, we describe a system developed in our laboratory, with which it is possible to perform biaxial Kerr magnetometry in an oblique field. Two components of the magnetization can be measured as a function of the magnitude and direction of the applied field. This technique is based on the MO Kerr effect. One of the in-plane components and the

perpendicular component of the magnetization are determined by measuring the transverse and the polar Kerr effect. The latter is characterized by a rotation of the plane of polarization; the amount of rotation is proportional to the component of the magnetization perpendicular to the film. The transverse effect involves a change in reflectivity of the light polarized parallel to the plane of incidence; this reflectivity change is linear with the change of the in-plane magnetization component perpendicular to the plane of incidence. By changing the angle of incidence and the state of polarization (SOP) of the incoming beam, it is possible to change the sensitivity for the perpendicular or the transverse component of the magnetization. This sensitivity and also the selectivity and the cross-talk characteristics of the measurement technique are discussed in Section 2.

Both the magnitude and the direction of the applied field can be changed. In order to change the field direction a rotatable sample holder was designed which makes use of two mirrors. In this way the sample can be rotated within the field of the magnet without changing the angle of incidence of the laser beam. More details of the sample holder and the rest of the measurement setup are given in Section 3. Although this is not the first time that mirrors have been used for an MO measurement system [8,9], as far as we know it is the first time that this setup has been used in order to measure the transverse Kerr effect.

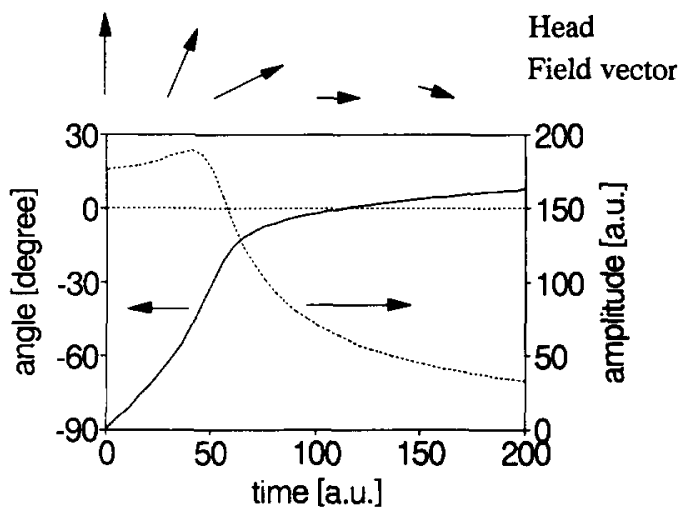

Fig. 2. Magnitude and direction of the single-pole-type head field versus time as experienced by a medium passing the write head. At $t=0$ the point of observation is positioned just under the head. At the top of the figure the complete vector of the head field is drawn for different $t$. 


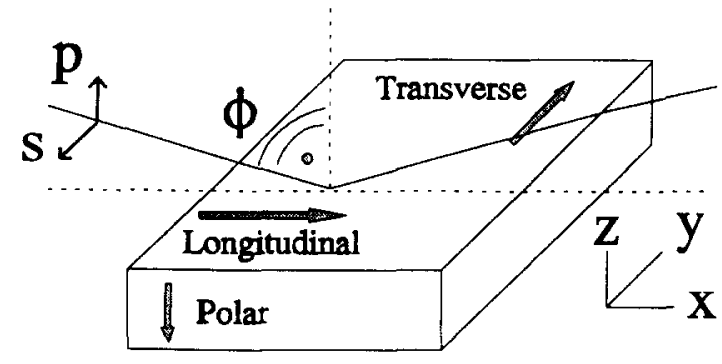

Fig. 3. Geometries for the transverse, longitudinal and polar Kerr effect (the shaded arrows define the direction of the magnetization for the different Kerr modes); definition of s-polarized (perpendicular to the plane of incidence) and p-polarized (parallel to the plane of incidence) light; definition of the coordinate axes.

In Section 4 the usefulness of the biaxial Kerr magnetometer is illustrated by presenting measurement results on rf-sputtered $\mathrm{Co}-\mathrm{Cr}$ films. These films, which have a perpendicular crystal anisotropy, are suitable candidates as a medium for perpendicular magnetic recording.

\section{Theory}

\subsection{Kerr effect}

Depending on the orientation of the magnetization towards the plane of incidence and the film plane different modes of the MO Kerr effect can be considered: In the transverse Kerr effect the magnetization is in the film plane perpendicular to the plane of incidence; in the longitudinal Kerr effect the magnetization lies in the film plane parallel to the plane of incidence; in the polar Kerr effect the magnetization is perpendicular towards the film plane (see Fig. 3).

The atomic origin of the Kerr effect is based on the combination of exchange and spin-orbit interactions [10]. These properties lead to the introduction of magnetization-dependent nondiagonal components in the dielectric tensor. For a homogeneously magnetized $\mathrm{Co}-\mathrm{Cr}$ film the effective dielectric tensor (MO Kerr effect) can be given by:

$\left(\begin{array}{ccc}\epsilon_{x x} & \epsilon_{x y} \cos (\gamma) & -\epsilon_{y z} \sin (\gamma) \sin (\beta) \\ \epsilon_{x y} \cos (\gamma) & \epsilon_{x x} & \epsilon_{y z} \sin (\gamma) \cos (\beta) \\ -\epsilon_{z y} \sin (\gamma) \sin (\beta) & \epsilon_{z y} \sin (\gamma) \cos (\beta) & \epsilon_{z z}\end{array}\right)$, where $\left(\epsilon_{z z}-\epsilon_{x x}\right)$ represent the optical anisotropy [12] and $\left(\epsilon_{y z}-\epsilon_{x y}, \epsilon_{y z}-\epsilon_{z y}\right)$ represent the magneto-optical anisotropy [13] (rf-sputtered $\mathrm{Co}-\mathrm{Cr}$ films have an hcp crystal structure with a strong $c$-axis texture perpendicular to the substrate [14]); $\gamma$ and $\beta$ define the direction of the magnetization towards the film normal $(=\gamma)$ and towards the plane of incidence $(=\beta)$.

For $(\beta, \gamma)=(0,90),(\beta, \gamma)=(90,90)$ and $(\beta, \gamma)=(X, 0)$ the matrix of Eq. (1) represents the dielectric tensors of the longitudinal, the transverse and the polar configuration, respectively. Starting with these dielectric properties and using the Maxwell equations, the geometrical parameters $\gamma, \beta$ and $\phi$ and general plane wave expressions, it is easy to calculate the Fresnel reflection coefficients [10]. They connect the incident $\left(=\bar{E}_{\text {inp }}\right)$ and reflected $\left(=\bar{E}_{\text {out }}\right)$ electric fields:

$\overline{E_{\text {out }}}=\left(\begin{array}{c}E_{\mathrm{p}, \text { out }} \\ E_{\mathrm{s}, \text { out }}\end{array}\right)=\left(\begin{array}{cc}r_{\mathrm{pp}} & r_{\mathrm{ps}} \\ r_{\mathrm{sp}} & r_{\mathrm{ss}}\end{array}\right)\left(\begin{array}{c}E_{\mathrm{p}, \text { inp }} \\ E_{\mathrm{s}, \text { inp }}\end{array}\right)=J_{\mathrm{CoCr}} \overline{E_{\mathrm{inp}}}$,

where $J_{\mathrm{CoCr}}$ is the Fresnel reflection matrix; $r_{\mathrm{pp}}, r_{\mathrm{ss}}$, $r_{\mathrm{ps}}$ and $r_{\mathrm{sp}}$ are the Fresnel reflection coefficients; and $\bar{E}_{\text {out }}$ and $\bar{E}_{\text {inp }}$ the complex vector amplitudes of the electric field of the EM-wave of the reflected and incident beam.

Neglecting the above mentioned (magneto)-optical anisotropies, i.e. assuming $\epsilon_{x x}=\epsilon_{z z}$ and $\epsilon_{x y}=$ $\epsilon_{y z}=\epsilon_{z y}$, the Fresnel coefficients for the thin, homogeneously magnetized film are [5]:

$$
\begin{aligned}
r_{\mathrm{pp}}= & \frac{n A-B}{n A+B} \\
& \times\left(1+\frac{\epsilon_{x y} \sin (2 \phi) \sin (\beta) \sin (\gamma)}{n^{2}\left(n^{2} \cos ^{2}(\phi)-1\right)+\sin ^{2}(\phi)}\right), \\
r_{\mathrm{ss}}= & \frac{A-n B}{A+n B}, \\
r_{\mathrm{ps}}= & -r_{\mathrm{sp}} \\
= & \frac{A \epsilon_{x y}}{n(n A+B)(A+n B)} \\
& \times\left[\cos (\gamma)+\sin (\gamma) \cos (\beta) \frac{\sin (\phi)}{n B}\right],
\end{aligned}
$$


with:

$A=\cos (\phi), \quad B=\sqrt{1-\frac{\sin ^{2}(\phi)}{n^{2}}} ;$

$\phi=$ the angle of incidence, $n=$ the complex refraction index.

From Eqs. (3)-(6) we can conclude:

- The longitudinal and perpendicular component of the magnetization will influence the nondiagonal component of the Fresnel matrix, while the transverse component of the magnetization will change the $r_{\mathrm{pp}}$. It is this difference which can be used to select the transverse component of the magnetization.

- As $\sin (\phi) /(n B)<1$ for most $\phi$, it can be concluded from the equations above that the contribution of the longitudinal component of the magnetization is smaller than that of the perpendicular component of the magnetization.

- Furthermore it can be concluded that for $\phi=0$ only the perpendicular component of the magnetization will contribute to the MO signal.

\subsection{Measurement principle}

In order to be able to change the field direction towards the sample a special sample holder was developed. The sample $(S)$ and two $\mathrm{Al}$ mirrors $\left(\mathrm{M}_{1}\right.$, $\mathrm{M}_{2}$ ) are positioned in a brass tube located between the pole shoes of a magnet (see Fig. 4). The tube can be rotated around the axis $\mathrm{AX}$, changing the field direction towards the film normal $\left(\gamma_{F}\right)$. Mirror $M_{1}$ turns the laser beam from the axis $\mathrm{AX}$ towards the sample surface. Another mirror $\mathbf{M}_{2}$ brings the laser beam back along the axis $\mathrm{AX}$. We chose for rotation of the sample holder over rotation of the magnet because of the compactness of this setup and the possibility to change to a larger magnet. a)

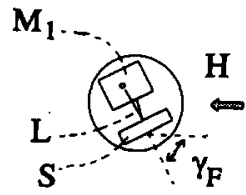

b)

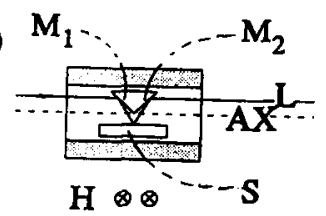

Fig. 4. Rotatable sample holder: (a) cross-section (front view), (b) cross-section (side view): $M_{1,2}=$ mirrors, $H=$ applied field, $L=$ light beam, $\gamma_{\mathbf{F}}=$ angle of the field, $\mathrm{AX}=$ rotation axis, $\mathrm{S}=$ sample, $\mathbf{G}=$ goniometer.
The perpendicular component of the magnetization is determined by measuring the polar Kerr effect. In this case $M_{1}$ and $M_{2}$ are formed by an aluminium prism with a top angle of $80^{\circ}$. This results in an angle of incidence on the sample that is smaller than $5^{\circ}$. The polarization direction is detected by measuring the intensity behind an analyzer which is almost crossed with the polarizer $\left(85^{\circ}\right)$. Best results (highest $\mathrm{S} / \mathrm{N}$ ratio) are obtained if the light to be analyzed is linearly polarized. For this reason, it is necessary to use incident light which is s- or ppolarized. Using intermediate modes will result in the introduction of a phase shift between both components and thus the introduction of an ellipticity in the reflected beam.

The transverse component of the magnetization is determined by measuring the transverse Kerr effect. Another aluminium prism with a top angle of $154^{\circ}$ is used. This results in an angle of incidence of $64^{\circ}$ on the sample. The incident light has to be linearly polarized $45^{\circ}$ towards the plane of incidence. As shown below, this will guarantee enough suppression of the sensitivity for the longitudinal and polar magnetization components. It also has the advantage that in this way a change of the p-Fresnel coefficient will result in a rotation of the polarization plane [15]. The phase shifts between the s- and p-components of the light at reflection with the aluminium mirrors and the $\mathrm{Co}-\mathrm{Cr}$ film is no problem because for this mirror configuration the total phase shift will be about $360^{\circ}$. Thus detection can be performed in the same way as for the polar case described above.

In the remaining part of this section we focus on the relation between the intensity measured by the detector and the polarization direction of the incident light beam in the case of the in-plane measurement mode. The selectivity of the measurement principle will also be discussed.

The reflection of the laser beam with the sampleholder and the Co-Cr film can be described by Jones algebra [16]:

$\overline{E_{\text {out }}}=J_{\text {anal }} J_{\mathrm{Al}} J_{\mathrm{CoCr}} J_{\mathrm{Al}} \overline{E_{\text {inp }}}$,

where $J_{\text {anal }}, J_{\mathrm{Al}}$ and $J_{\mathrm{CoCr}}$ are the Jones matrices of the analyzer, the reflection with the $\mathrm{Al}$ mirror and the reflection with the $\mathrm{Co}-\mathrm{Cr}$ sample, respectively, and $\bar{E}_{\text {inp }}$ and $\bar{E}_{\text {out }}$ are the complex field amplitude vectors of the incident and reflected light beam. 
Assuming that the incident light is linearly polarized under an angle of $\xi$ degrees with the p-direction, $\bar{E}_{\text {inp }}$ has the form:

$\overline{E_{\text {inp }}}=E_{0}\left(\begin{array}{c}\cos (\xi) \\ \sin (\xi)\end{array}\right)$,

where $E_{0}$ is a complex constant including the intensity of the incident light beam.

Using Eqs. (7) and (8) the electric field of the reflected light beam, $\bar{E}_{\text {out }}$, can be calculated:

$$
\begin{aligned}
& \overline{E_{\text {out }}}=\left(\begin{array}{c}
E_{\mathrm{p}, \text { out }} \\
E_{\mathrm{s}, \text { out }}
\end{array}\right)
\end{aligned}
$$

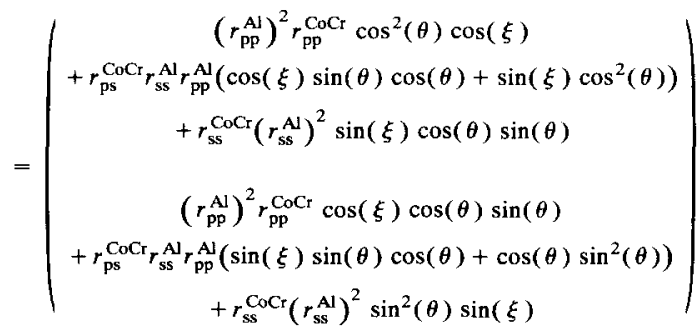

$$
\begin{aligned}
& =\left(\begin{array}{c}
\left(r_{\mathrm{pp}}^{\mathrm{Al}}\right)^{2} r_{\mathrm{pp}}^{\mathrm{CoCr}} Q_{1} \\
+r_{\mathrm{ps}}^{\mathrm{CoC}} r_{\mathrm{ss}}^{\mathrm{Al}} r_{\mathrm{pp}}^{\mathrm{Al}} Q_{2} \\
+r_{\mathrm{ss}}^{\mathrm{CoCr}}\left(r_{\mathrm{ss}}^{\mathrm{Al}}\right)^{2} Q_{3} \\
\left(r_{\mathrm{pp}}^{\mathrm{Al}}\right)^{2} r_{\mathrm{pp}}^{\mathrm{CoCr}} Q_{4} \\
+r_{\mathrm{ps}}^{\mathrm{CoCC}} r_{\mathrm{ss}}^{\mathrm{Al}} r_{\mathrm{pp}}^{\mathrm{Al}} Q_{5} \\
+r_{\mathrm{ss}}^{\mathrm{CoC}}\left(r_{\mathrm{ss}}^{\mathrm{Al}}\right)^{2} Q_{6}
\end{array}\right)
\end{aligned}
$$

where $\xi=$ the polarization direction of the incident beam towards the plane of incidence; $\theta=$ the analyzer direction towards the plane of incidence; $Q_{1}$, $Q_{2}, \ldots Q_{6}$ are constants that depend on $\theta$ and $\xi$; $r_{\mathrm{pp}}^{\mathrm{Al}}, r_{\mathrm{ss}}^{\mathrm{Al}}$ are the Fresnel reflection coefficients of the aluminium mirrors; $r_{\mathrm{pp}}^{\mathrm{CoCr}}, r_{\mathrm{ss}}^{\mathrm{CoCr}}, r_{\mathrm{ps}}^{\mathrm{CoCr}}=r_{\mathrm{sp}}^{\mathrm{CoCr}}$ the Fresnel coefficients of the sample. These coefficients can be calculated using Eqs. (3)-(6).

From Eq. (9) it can be concluded that the sensitivity for perpendicular and longitudinal components of the magnetization can be suppressed by choosing $\xi$ and $\theta$ so that the terms with $r_{\mathrm{ps}}$ are close to zero. In Fig. 5 we plot the constants $Q_{1}, Q_{2}, \ldots Q_{6}$ (see Eq. (9)) as a function of $\xi$ under the assumption $\theta=\xi$ +85 (analyser $85^{\circ}$ crossed with polarizer). From this plot it can be concluded that the terms with $r_{\mathrm{ps}}^{\mathrm{CoCr}}$ $\left(Q_{2}\right.$ and $\left.Q_{5}\right)$ are small for $\xi= \pm 48.3^{\circ}$, while the terms with $r_{\mathrm{pp}}^{\mathrm{CoCr}}$ are large around this angle. This

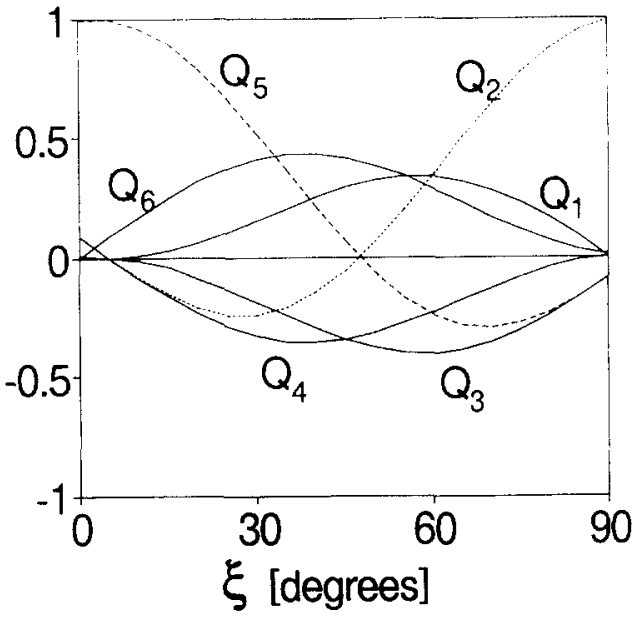

Fig. 5. The constants $Q_{1}, Q_{2}, \ldots Q_{6}$ as a function of the polarization direction of the incident light beam $\left(\theta=\xi+85^{\circ}\right)$.

guarantees the desired selectivity of the measurement mode.

Using the optical properties of $\mathrm{Co}-\mathrm{Cr}$ as measured by ellipsometry $\left(n^{\mathrm{CoCr}}=2.12-4.2 \mathrm{j}, \epsilon_{x y}^{\mathrm{CoCr}}=\right.$ $-0.144+0.062 \mathrm{j}[16])$, the optical properties of $\mathrm{Al}$ as found in literature $\left(n^{\mathrm{Al}}=1.304-7.4 \mathrm{j}\right.$ [12] $)$, the geometrical parameters of the aluminium prism and Eqs. (3), (5) and (6), the Fresnel reflection matrices $J_{\mathrm{Al}}$ and $J_{\mathrm{CoCr}}$ were calculated. These values were substituted in Eq. (9) which was converted in an intensity expression. The maximum relative intensity change as induced by the perpendicular or the transverse component of the magnetization are plotted in Fig. 6 as a function of the polarization direction of the incident light. For $\xi=48-48.66^{\circ}$ the cross-talk of the perpendicular component is smaller than -20 dB. From Eq. (5) it appears that the cross-talk of the longitudinal component is also small.

\section{Measurement system}

The direction of the plane of polarization was measured by the intensity technique as described, for example, in Ref. [17]. Two analyzers were used, which were placed under $85^{\circ}$ and $-85^{\circ}$ towards the polarizer direction (see Fig. 7) in order to suppress light source noise. With a normal beam splitter, half of the light was deflected in the second detector. In 


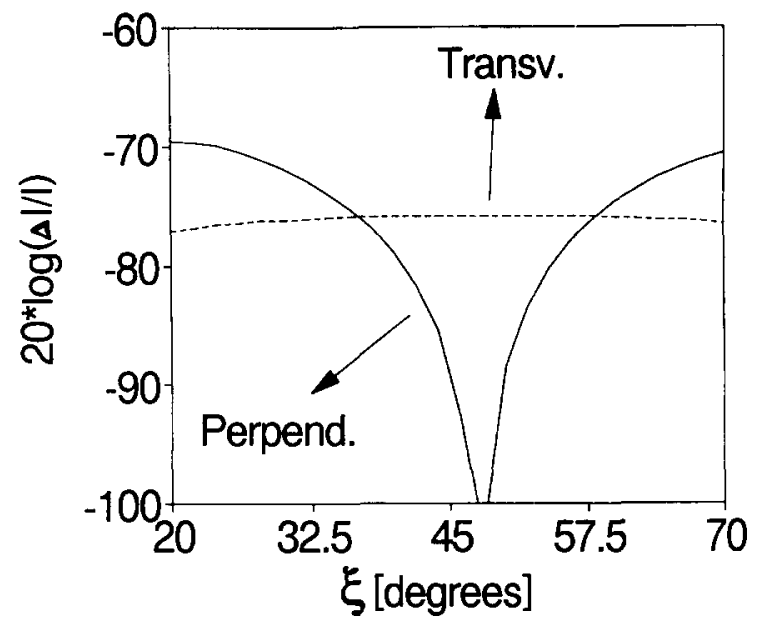

Fig. 6. Relative intensity change as induced by perpendicular (solid) and transverse (dashed) component of the magnetization as a function of the polarization direction of the incident light beam.

order to guarantee linearly polarized light falling on both analyzers, the directions of the eigenmodes of the rotatable sample holder, of the beam splitter and of the analyzer had to be aligned. For this reason a special beam splitter holder was designed. Analyzer 2, detector 2 and the beam splitter (see Fig. 7) are integrated in one optical device (see Fig. 8). Before each measurement the polarizer and beam splitter were manipulated in order to create a system in which linearly polarized light could propagate invariantly.

The selectivity of the measurement method was checked by cross-talk experiments on sputtered Co$\mathrm{Cr}$ films. Using the perpendicular mirror set, the

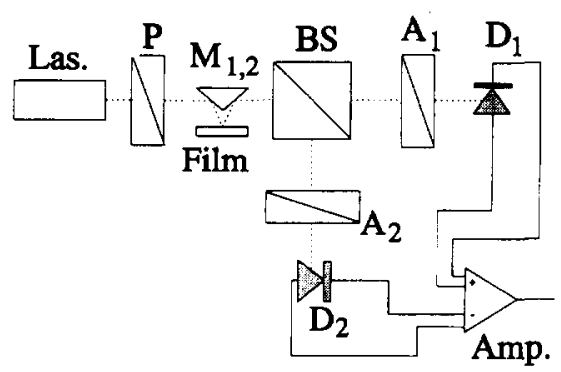

Fig. 7. Measurement setup magneto-optical system (the dashed line represents the laser light beam): Las $=$ laser, $P=$ polarizer, $M_{1} \quad M_{2}=$ mirrors, $B S=$ beam splitter, $A_{1}, A_{2}=$ analyzers, $P_{1}$ $P_{2}=$ polarizers, $D_{1}, D_{2}=S i$ detectors, Amp $=$ differential amplifier.

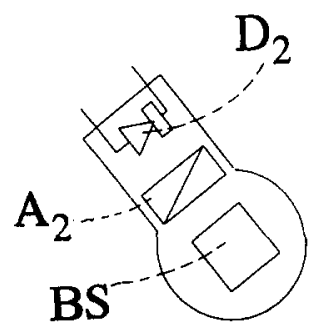

Fig. 8. Rotatable beam-splitter holder: schematic drawing of the rotating part of the holder: $\mathbf{A}_{2}=$ analyzer, $\mathbf{B S}=$ beam splitter, $D_{2}=$ detector (see also Fig. 7).

hysteresis curves were measured at $\gamma_{\mathrm{F}}=90^{\circ}$ and $0^{\circ}$. The signal amplitude of the former was smaller than $1 \%$ of the signal amplitude of the latter, which guarantees reliable measurement results over the complete range of field angles. The cross-talk figure for $\gamma_{\mathrm{F}}=0^{\circ}$ was about $8 \%$ of the signal amplitude at $\gamma_{\mathrm{F}}=90^{\circ}\left(\xi=(45 \pm 0.5)^{\circ}\right)$. This result is in agreement with theory. Better results can be obtained by a more accurate alignment of the $\xi$.

Although the transverse Kerr effect is maximum around $85^{\circ}$, the maximum practical angle of incidence was about $65^{\circ}$. For this reason the signal for the in-plane measurements was more than 5 times weaker than in the signal in the perpendicular measurements.

\section{Results and discussion}

The above described measurement system was used to investigate an rf-sputtered $\mathrm{Co}_{81} \mathrm{Cr}_{19}$ film (thickness $=46 \mathrm{~nm}, \quad M_{\mathrm{s}}=442 \mathrm{kA} / \mathrm{m}, \quad K_{1}=94$ $\mathrm{kJ} / \mathrm{m}^{3}$, hcp texture better than $5^{\circ}$, columnar structure, column width $<40 \mathrm{~nm}$ ) [14]. Although the film has a $Q$-factor $\left(=2 K_{1} / \mathrm{u}_{0} M_{\mathrm{s}}^{2}\right)$ of 0.76 , the existence of domains will guarantee that the magnetization is perpendicular to the film substrate in zero field; only in the areas between neighbouring Weiss regions near the surface and near substrate interface will the spins have a nonperpendicular orientation $[18,19]$. Since the penetration depth of the light is about half the film thickness, the measurement results presented here are bulk values. Some typical curves measured at different field angles are presented in Fig. 9. In these curves the perpendicular component of the magnetization is plotted as a func- 


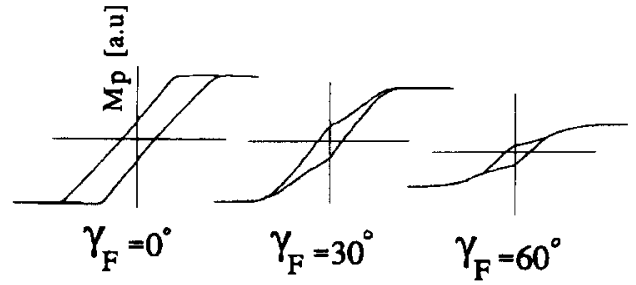

Fig. 9. Perpendicular component of the magnetization $\left(=M_{\mathrm{p}}\right)$ as a function of applied field for different angles of the field.

tion of the magnitude of the applied field in the $\gamma_{F}$ direction.

The in-plane results showed no clear field-angle dependence. The small hysteresis disappeared completely for angles smaller than $80^{\circ}$. In order to check the measurement results, biaxial VSM measurements were performed on the same sample; similar results were obtained.

In order to be able to interpret the measured data we suggest a magnetization distribution as shown in Fig. 10. This means that the film contains magnetic domains which are magnetized in more or less opposite directions. The magnetization within the domains is rotated towards the field vector. It is assumed that the rotation $(=\gamma)$ is equal for both up and down magnetized domains. This is a reasonable assumption because the in-plane component of the field, which is responsible for the rotation, is equal for both types of domains.

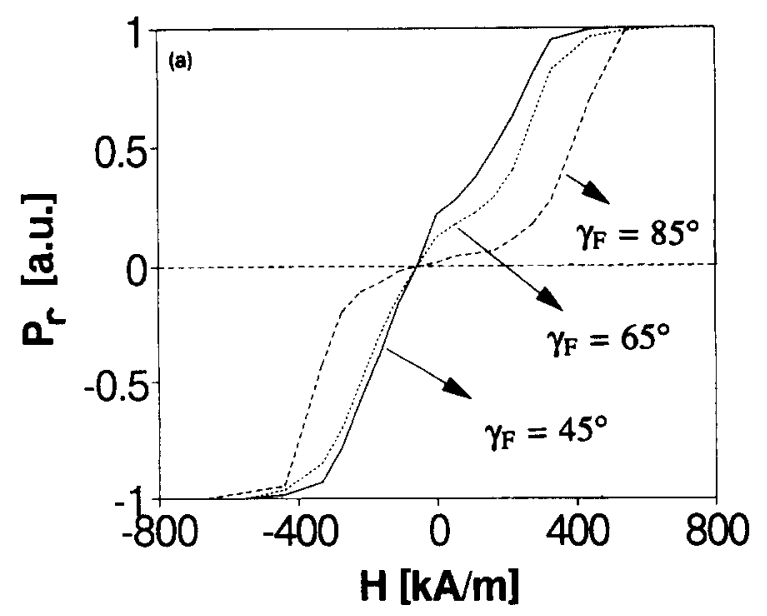

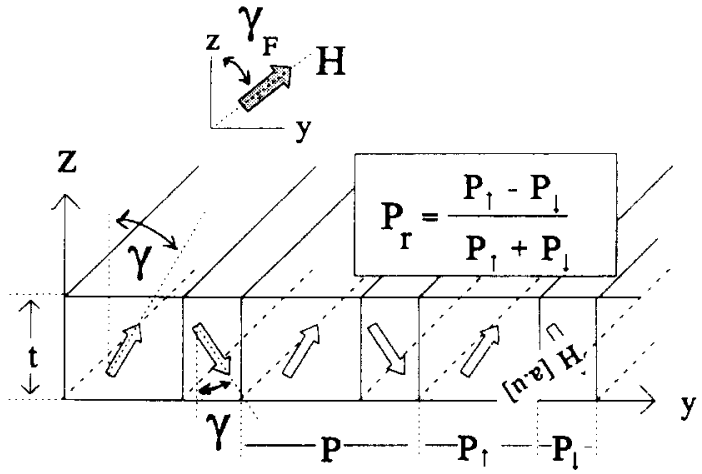

Fig. 10. Model of the distribution of the magnetization in an oblique field.

Assuming the configuration of Fig. 10, the following relations hold:

$M_{\mathrm{s}} \sin (\gamma)=M_{\mathrm{i}}$

$M_{\mathrm{s}} \frac{P_{\uparrow}-P_{\downarrow}}{P_{\uparrow}+P_{\downarrow}} \cos (\gamma)=P_{\mathrm{r}} \cos (\gamma) M_{\mathrm{s}}=M_{\mathrm{p}}$,

where $M_{\mathrm{i}}, M_{\mathrm{p}}$ are the in-plane and perpendicular components of the average magnetization vector, respectively, $M_{\mathrm{s}}$ is the saturation magnetization, $\gamma$ is the angle between the magnetization and the easy direction, and $P_{\mathrm{r}}, P_{\uparrow}$ and $P_{\downarrow}$ are as defined in Fig. 10. The factor $P_{\mathrm{I}}$, the phase fraction, quantifies the

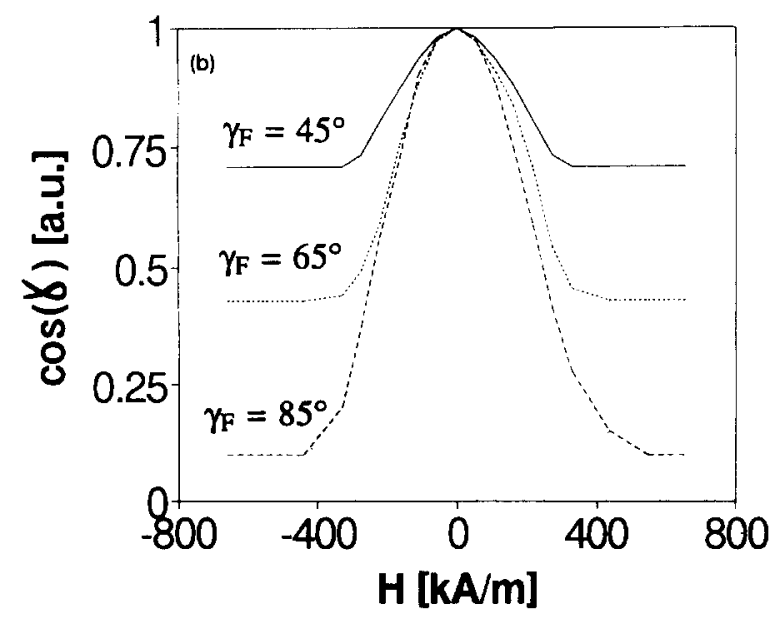

Fig. 11. Calculated (a) relative domain pattern and (b) relative rotation contribution to the magnetization reversal as a function of the applied field $(=H)$ for different angles of the field $\left(=\gamma_{\mathrm{F}}\right)$. 
influence of the domain pattern on the perpendicular component of the magnetization. The domain pattern can change by domain wall motion or by the nucleation of reversal domains. The factor $\cos (\gamma)$ illustrates the influence of the rotation of the magnetic spins on the average perpendicular component of the magnetization.

These parameters as calculated from the measured data, are shown in Fig. 11 for different field directions. The curves for $P_{\mathrm{r}}$ (Fig. 11a) vary between -1 and $1\left(=M_{\mathrm{s}}\right.$ and $-M_{\mathrm{s}}$ saturated). The curves for $\cos (\gamma)$ (Fig. 11b) vary between $\cos \left(\gamma_{\mathrm{F}}\right)$ and 1 . These results suggest that the angle of the magnetization is zero for a zero applied field, which is in agreement with the results of torque magnetometer measurements on the same sample [14].

From Eq. (11) it is possible to calculate the partial contributions to the reversal of the perpendicular magnetization. Taking the derivative of (11) on both sides gives:

$$
\begin{aligned}
\frac{\mathrm{d} M_{\mathrm{p}}}{\mathrm{d} H} & =\frac{\partial P_{\mathrm{r}}}{\partial H} \cos (\gamma) M_{\mathrm{s}}+\frac{\partial \cos (\gamma)}{\partial H} P_{\mathrm{r}} M_{\mathrm{s}} \\
& =A M_{\mathrm{s}}+B M_{\mathrm{s}}
\end{aligned}
$$

where $A$ is the contribution of the change in phase fraction to the magnetic reversal, and $B$ is the contribution of the rotation to the magnetic reversal.

The constant $B$ appears to be negative in the first and the third quadrants, and slightly positive in the second and fourth quadrants of the hysteresis curve. Thus, for this type of $\mathrm{CoCr}$ film, the rotation will usually give a negative contribution (counteracting the magnetic reversal process) to the change in the average perpendicular magnetization. This conclusion is in agreement with a monotonically increasing perpendicular hysteresis curve. The factor $A$ appears to be positive for all fields. For small fields its value is nonzero; from this we can conclude that the reversal of the magnetization at small fields is mainly done by changing the phase fraction of the domain pattern.

For smaller angles of the field the factor $B$ becomes smaller. However, this effect can not be considered as a measurement result, since it is implied by our assumptions in the model of Fig. 10 (since the magnetization in both types of domains are parallel, the in-plane component at $H=0$ must be zero for symmetry reasons).

The sudden change in the differential susceptibility of the perpendicular hysteresis curve can be partly explained by the sign change of the factor $B$ at $H=0$. However, the fact that we do not observe a similar kink for $H=-H_{c}$ (also a sign change of $B$ ) makes it clear that this is not the complete explanation. Energy calculations have to be performed in order to explain this point.

\section{Conclusions and final remarks}

A biaxial Kerr magnetometer based on the perpendicular and transverse Kerr effect was developed in our laboratory. By the use of an Al mirror set it was possible to change the direction of the applied field and to perform angle-dependent measurements. By paying careful attention to the geometry of the sample holder, the cross-talk figures could be kept small and the simple intensity technique could be used to measure the Kerr rotation. The measurement system was checked by comparing the results with biaxial VSM measurement results on a $\mathrm{CoCr}$ sample. Good agreement was obtained. The preliminary results suggest that in this type of $\mathrm{Co}-\mathrm{Cr}$ film the reversal of the average perpendicular magnetization is opposed by the rotation of the spins. The change of the phase fraction is mainly responsible for the reversal process at small fields.

The major advantage of a biaxial Kerr magnetometer over a biaxial VSM is its high (equal to the sensitivity of the anomalous Hall technique) and uniform (position-independent) [20] sensitivity. This makes this technique very interesting for anisotropy studies of ultrathin films. Furthermore, because of the surface sensitivity of the Kerr effect (the penetration depth of $\mathrm{He}-\mathrm{Ne}$ light in the transition metals is about $15 \mathrm{~nm}$ ) it is a suitable technique for studying high-density recording materials [18]. However, it should be mentioned here that Kerr magnetometry is an indirect method; i.e. it is not the average magnetic moment but the effective dielectric tensor that is determined. For inhomogeneous systems the relation between these two physical quantities can be a nonlinear one [21]. 


\section{Acknowledgements}

Dr Jan Bernards and Dr Hugo Cramer of the Philips Laboratories in Eindhoven, must be thanked for performing biaxial VSM experiments on our samples. The authors would also like to thank Professor U. Enz for fruitful discussions and his stimulating comments.

\section{References}

[1] S. Iwasaki and Y. Nagamura, IEEE Trans. Magn. 13 (1977) 1272.

[2] J.C. Mallinson and H.N. Bertram, IEEE Trans. Magn. 20 (1984) 461.

[3] K. Ozawa and M. Takashi, IEEE Trans. Magn. 19 (1983) 1635.

[4] J.P.C. Bernards, G.J.P. van Engelen, C.P.G. Schrauwen, H.A.J. Cramer and S.B. Luitjens, IEEE Trans. Magn. 26 (1990) 216.

[5] J.M. Florczak and E. Dan Dahlberg, J. Appl. Phys. 67 (1990) 7520.
[6] S. de Haan, J.C. Lodder and Th. J.A. Popma, J. Magn. Soc. Jpn. Oct. 15-S2 (1991) 349.

[7] M. Abe, M. Gomi, K. Shono, Y. Mori and S. Nomura, Jpn. J. Appl. Phys. 16 (1977) 279.

[8] R.M.A. Azzam and N.M. Bashara, Ellipsometry and Polarized Light (North-Holland, Amsterdam, 1977).

[9] K. Hemmes, W. Lisowski, J.C. Lodder, L.J. Hanekamp and Th.J.A. Popma, J. Phys. D: Appl. Phys. 19 (1986) 1311.

[10] P.N. Argyres, Phys. Rev, 97 (1955) 334.

[11] M.J. Freiser, IEEE Trans. Magn. 4 (1968) 152.

[12] Handbook of Chemistry and Physics, 66th edn. (1985-86) E-376.

[13] E.A. Gan'shina, G.S. Krinchik, L.S. Mironova and A.S. Tablin, Sov. Phys. JETP 51 (1980) 369.

[14] W.J.M.A. Geerts, J.G.Th. te Lintelo, J.C. Lodder and Th.J.A. Popma, IEEE Trans. Magn. 26 (1990) 36.

[15] D.B. Dove, J. Appl. Phys. 34 (1963) 2067.

[16] W.J.M.A. Geerts, J.C. Lodder and Th.J.A. Popma, Rev. Sci. Instrum. 63 (1992) 1805.

[17] D.J. Mapps, N. Mahvan and M.A. Akhter, IEEE Trans. Magn. 25 (1989) 4192.

[18] W.J.M.A. Geerts, J.C. Lodder and Th.J.A. Popma, J. Magn. Magn. Mater. 104-107 (1992) 971.

[19] A. Hubert, IEEE Trans. Magn. 21 (1985) 1604.

[20] A. Zieba and S. Foner, Rev. Sci. Instrum. 53 (1982) 1344.

[21] P.G. van Engen, PhD Thesis, Delft University of Technology, The Netherlands, 1991. 